\title{
Quantitative point-of-care colorimetric assay modelling using a handheld colorimeter
}

\author{
Kristen Abels ${ }^{\dagger}$, Elizabeth M. Salvo-Halloran§, Dawn White§, Monsur Ali§, Nisha R. Agarwal \$\#, \\ Vincent Leung ${ }^{\dagger}$, Muntakim Ali ${ }^{\dagger}$, Mariam Sidawi ${ }^{\dagger}$, Alfredo Capretta§, John D. Brennan§, Jake Nease*† \\ and Carlos D. M. Filipe*广
}

†Department of Chemical Engineering and \$Biointerfaces Institute, McMaster University, 1280 Main St. West, Hamilton, ON L8S 4M1, Canada. "Nano-Imaging and Spectroscopy Laboratory, Faculty of Science, University of Ontario Institute of Technology, 2000 Simcoe Street North, Oshawa, Ontario L1G 0C5, Canada.

\section{COLORIMETRIC ASSAY MODELLING}

All colorimetric assay modelling was performed via minimization of the sum of squared error between the model and experimental data points. In all cases, one function that takes in the analyte concentration and outputs the estimated absorbance value at the wavelength of interest is derived from first principles. A calibration curve is first determined for each colorimetric assay (whereby the experimentally measured absorbance of solutions of known concentration are plotted against the property of interest to determine the sum of squared error model parameters), after which said model may be used for property prediction based off of $\mathrm{Nix}^{\mathrm{TM}}$ color measurements.

Determination of model parameters was performed in MATLAB using the Curve Fitting Tool. For analyte property prediction based off of $\mathrm{Nix}^{\mathrm{TM}}$ color measurements, a sum of squared error minimization approach was used for the more complex $\mathrm{pH}$ model, whereas a simple calculation of the model inverse function was used for the enzymatic assays.

In all cases, the Scott Burns algorithm was used to convert the Nix ${ }^{\mathrm{TM}}$ sensor CIELAB color coordinates into an estimated absorb ance spectrum for analysis. ${ }^{1}$ Initial and final sample measurements were obtained before and after performing each assay so that the initial background absorbance could be subtracted as a way of standardizing the data and eliminating the impact of background color. Each assay model is described in further detail in the proceeding sections.

\section{CUSTOM WELL PLATE}

A custom white acetal plate with well diameters that perfectly matched the opening of the Nix ${ }^{\mathrm{TM}}$ Color Sensor was produced to ensure that there was no light interference during the color measurement. The plate contained a pattern of $5 \times 11$ wells and had dimensions as follows: Length x Width x Depth: 300 x 150 x 16 mm; Well Inner Diameter: 6.38 mm; Well Outer Diameter: 7.35 mm; Depth: 8 mm; Volume of $1 \mathrm{ml}$; Height and Width of lip around well: $3 \mathrm{~mm}, 0.97 \mathrm{~mm}$; Inter-well spacing (center to center): $25 \mathrm{~mm}$.

The custom well-plate not only blocked ambient light but also ensured consistent measurement conditions (e.g. distance from colorimeter to sample) throughout the experiments, which was important for consistent absorbance spectra estimation across samples.

\section{PH ASSAY}

The universal indicator used to estimate $\mathrm{pH}$ of a solution is comprised a variety of $\mathrm{pH}$ indicators: methyl red, methyl orange, bromophenol blue and phenolphthalein. Each contributes to the solution color based on the degree of ionization. As such, the Henderson-Hasselbach equation can be used to determine the relative proportion of each component indicators' conjugate acid-base pair for any given $\mathrm{pH}$. Using the approximated absorbance spectra for each indicator in acid and conjugate base form, the resultant absorbance was taken to be the linear combination of the absorbances from each component, weighted by their respective proportion in solution.

For each indicator, $\frac{\left[\mathrm{I}^{-}\right]}{[\mathrm{HI}]}=10^{\mathrm{pH}-\mathrm{pKa}}$, so $x_{H I}=\frac{X_{\text {indicator }}}{1+10^{\mathrm{pH}-\mathrm{pKa}}}$ and $\mathrm{x}_{\mathrm{I}^{-}}=x_{\text {indicator }}-x_{H I}$ 
Then

This can be further written as

$$
A b s_{\text {resultant }}=\sum_{\text {indicators }} x_{H I} A b s_{H I}+x_{I^{-}} A b s_{I^{-}}
$$

Grouping the constants,

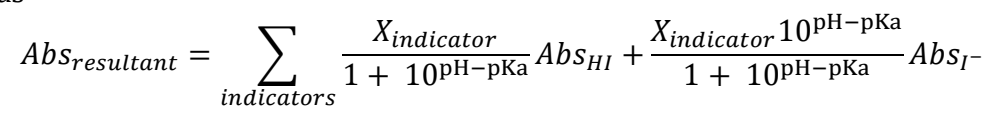

$$
A b s_{\text {resultant }}=\sum_{i}\left(\frac{a_{i}}{1+10^{\mathrm{pH}-\mathrm{c}_{\mathrm{i}}}}+\frac{b_{i} 10^{\mathrm{pH}-\mathrm{c}_{\mathrm{i}}}}{1+10^{\mathrm{pH}-\mathrm{c}_{\mathrm{i}}}}\right)
$$

Since different indicators have different maximum absorbance wavelengths (resulting in changes in both solution hue and intensity as a function of $\mathrm{pH}$ ), rather than considering one wavelength only, multiple wavelength ranges were considered for $\mathrm{pH}$ modelling and prediction. Scott Burns' algorithm for absorbance spectrum estimation provides estimated absorbance values in 10 nm increments over the visible light spectrum. In this case, the summed absorbance values over the range of 500-550 $\mathrm{nm}$ and 550-600 $\mathrm{nm}$ were considered, as these ranges were found to provide sufficient information for $\mathrm{pH}$ prediction in the $\mathrm{pH}$ range studied. In these two ranges, a maximum of three inflection points were observed over the range of interest. For this reason, it was decided to fit the curves with the model in Equation (S1) above with three indicators using the MATLAB Curve Fitting Tool.

\section{Table S1. Calibration curve parameters for solution pH based on estimated absorbance values from CIELAB} measurements.

\begin{tabular}{|l|l|l|}
\hline Parameter & $500-550 \mathrm{~nm}$ Model & $550-600 \mathrm{~nm}$ Model \\
\hline $\mathrm{a}_{1}$ & 1.152 & 0.8313 \\
\hline $\mathrm{b}_{1}$ & 0.000 & 0.000 \\
\hline $\mathrm{c}_{1}$ & 4.624 & 4.107 \\
\hline $\mathrm{a}_{2}$ & 0.000 & 0.000 \\
\hline $\mathrm{b}_{2}$ & 0.9723 & 1.133 \\
\hline $\mathrm{c}_{2}$ & 5.087 & 5.119 \\
\hline $\mathrm{a}_{3}$ & 0.000 & 0.000124 \\
\hline $\mathrm{b}_{3}$ & 0.3035 & 0.07704 \\
\hline $\mathrm{c}_{3}$ & 8.546 & 8.774 \\
\hline $\mathrm{R}^{2}$ & 0.9449 & 0.9602 \\
\hline
\end{tabular}

The ability to predict solution pH based off of the two calibration curves was assessed by determining the pH in the range of $2-12$ to the nearest $0.01 \mathrm{pH}$ units that results in the lowest sum of squared residuals - that is, the minimum sum of squared differences between sample measurements and calibration curve values for the two wavelength ranges (500-550 $\mathrm{nm}$ and 550-600 $\mathrm{nm}$ ) (Equation S2).

$$
\min _{p H}\left[\left(a b s_{500-550}-\widehat{a b} s_{500-550}(p H)\right)^{2}+\left(a b s_{550-600}-\widehat{a b s} s_{550-600}(p H)\right)^{2}\right]
$$

Even though the summed 500-550 nm absorbance measurement for a sample may intersect the calibration curve at more than one $\mathrm{pH}$, the corresponding summed 550-600 nm absorbance measurement provides the additional information required for a unique $\mathrm{pH}$ estimate to be determined (and vice-versa). The predicted $\mathrm{pH}$ of the colourless solutions used to generate the calibration curves was found to be quite accurate over the entire range of study with $\mathrm{R}_{\text {train }^{2}}=0.984$. The yellow-tinted solutions (shown in Figure S1) were found to have lower prediction accuracy with $\mathrm{R}_{\text {test }}{ }^{2}=0.884$, but still significantly higher than the prediction accuracy obtained with the CIELAB linear-in-parameters regression (Equation S3). In this case, the ability to subtract the initial solution absorbance from the final absorbance with indicator is crucial to accurate prediction.

$$
\hat{y}=a_{0}+a_{1} L+a_{2} A+a_{3} B+a_{4} L A+a_{5} L B+a_{6} A B+a_{7} L^{2}+a_{8} A^{2}+a_{9} B^{2}+a_{10} \log (\mathrm{B}+50)
$$


Table S2. Linear-in-parameters basis function regression for solution pH calibration curve development based on CIELAB values.

\begin{tabular}{|l|c|}
\hline Parameter & Value \\
\hline $\mathrm{a}_{0}$ & -136.7419 \\
\hline $\mathrm{a}_{1}$ & -0.9237 \\
\hline $\mathrm{a}_{2}$ & -0.1374 \\
\hline $\mathrm{a}_{3}$ & -0.6072 \\
\hline $\mathrm{a}_{4}$ & 0.0074 \\
\hline $\mathrm{a}_{5}$ & -0.0138 \\
\hline $\mathrm{a}_{6}$ & -0.0052 \\
\hline $\mathrm{a}_{7}$ & 0.0196 \\
\hline $\mathrm{a}_{8}$ & -0.0009 \\
\hline $\mathrm{a}_{9}$ & 0.0105 \\
\hline $\mathrm{a}_{10}$ & 39.8772 \\
\hline $\mathrm{R}_{\text {train }}{ }^{2}$ & 0.998 \\
\hline $\mathrm{R}_{\text {test }}{ }^{2}$ & 0.749 \\
\hline
\end{tabular}

It was found that this model fit the training data (colourless solutions) very well $\left(\mathrm{R}_{\text {train }^{2}}=0.998\right)$ but did not fit the testing set $(\mathrm{yellow}$ tinted solutions) well at all $\left(\mathrm{R}_{\mathrm{test}^{2}}{ }^{2}=0.749\right)$, demonstrating that the estimated absorbance method is superior due to its ability to account for initial solution colour.

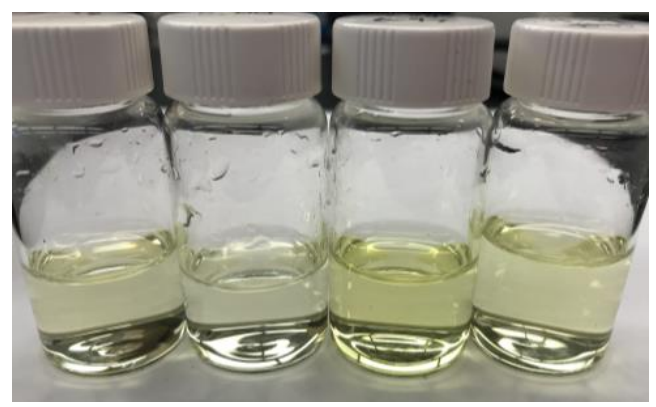

Figure S1. Examples of the solutions tinted yellow with tartrazine (pH 3, 5, 7 and 10).

\section{DNA GOLD NANOPARTICLE ASSAY}

The DNA-Crosslinked AuNP fabrication procedure used herein was based on previous studies. ${ }^{2-3} 100 \mu \mathrm{L}$ of $100 \mu \mathrm{M}$ S1 and S2 thiolmodified DNA (see Table S3 for sequences obtained from Integrated DNA Technologies (IDT)) were separately added to solutions of $3 \mathrm{~mL} 13 \mathrm{~nm}$ citrate-capped gold nanoparticles $(\sim 10 \mathrm{nM})$ with $1.9 \mathrm{~mL}$ milliQ water added to obtain a final volume of $5.0 \mathrm{~mL}$ each. Following 24-hour incubation at room temperature, $50 \mu \mathrm{L}$ Tris-HCL (1 M, pH 7.5) and $450 \mu \mathrm{L}$ aqueous NaCl (1M) were added to each solution followed by incubation at room temperature for another 24 hours. $25 \mu \mathrm{L}$ Tris-HCL (1M, pH 8.3) and $250 \mu \mathrm{L}$ aqueous NaCl (1M) were then added to each DNA-AuNP solution, followed by a final incubation at room temperature 24 hours. Each mixture was then divided into $\sim 550 \mu \mathrm{L}$ volumes and centrifuged at $4^{\circ} \mathrm{C}(14000 \mathrm{rpm}, \sim 21000 \mathrm{~g})$ for $20 \mathrm{~min}$. The supernatant was discard ed and replaced with $250 \mu \mathrm{L}$ Tris-HCl buffer $(20 \mathrm{mM}$, pH 7.5) to obtain the DNA-modified AuNPs at a concentration of $\sim 12 \mathrm{nM}$.

DNA-Crosslinked AuNP aggregates were then prepared by mixing $50 \mu \mathrm{L}$ of the S1 and S2-modified AuNPs, heating at $70{ }^{\circ} \mathrm{C}$ for two minutes following by overnight storage in the fridge at $4{ }^{\circ} \mathrm{C}$. Twenty-four hours later, the DNA-Crosslinked AuNP solutions were centrifuged at 14,000 rpm for 5 minutes and the supernatant was removed. The resulted DNA-Crosslinked AuNP pellets were used in the assays. Figure S2 shows a schematic of the assay. 
A DNA-Crosslinked AuNP Preparation

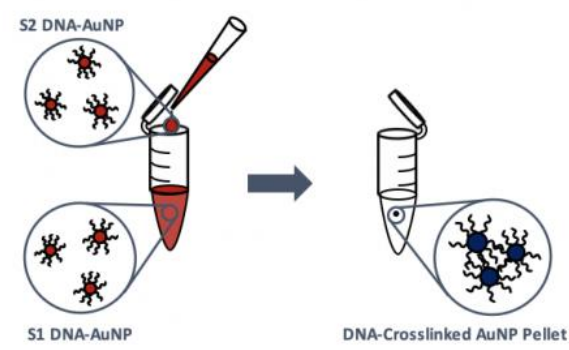

B DNA-Crosslinked AuNP + DNase I
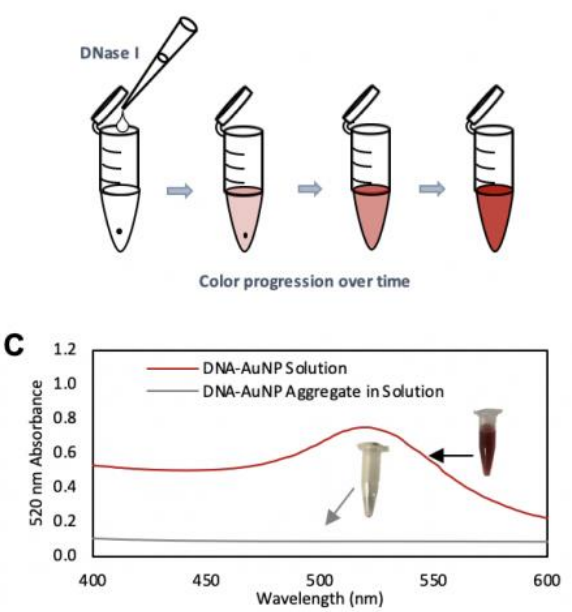

Figure S2 A) Schematic of the DNA-Crosslinked AuNP preparation. B) Schematic depicting the assay procedure. C) Comparison of absorbance spectrum of DNA-Crosslinked AuNP pellet compared to the un-crosslinked AuNP, measured with the Tecan M200 Pro Plate Reader.

Table S3. DNA sequences obtained from IDT for DNA-crosslinked gold nanoparticle synthesis.

\begin{tabular}{|l|l|}
\hline Sequence S1 & 5' - CAA GCT TGC CAT CAT GTC GAT CTT TTT TTT TT/3ThioMC3-D/ - 3' \\
\hline Sequence S2 & 5' - GAT CGA CAT GAT GGC AAG CTT GTT TTT TTT TT/3ThioMC3-D/ - 3' \\
\hline
\end{tabular}

Equation (S4) was used for modelling the sigmoidal trends in absorbance over varying DNase I concentration and time:

$$
A_{520}([\text { DNase I }])=\frac{a}{\left(1+e^{-b([\text { DNase I }] t-c)}\right)}
$$

Given $\mathrm{Nix}^{\mathrm{TM}}$ data collected after 25 minutes of incubation of the DNase I samples with DNA-Crosslinked AuNP pellets, the following estimated absorbance curve at $520 \mathrm{~nm}$ was obtained, shown plotted against DNase I concentration. The model parameters were again determined using the MATLAB Curve Fitting Tool and were found to be $\mathrm{a}=0.1514$ and $\mathrm{b}=558.8$, and $\mathrm{c}=0.005184$ with $\mathrm{R}^{2}=$ 0.9748. Considering the Tecan M200 Pro Plate Reader $520 \mathrm{~nm}$ absorbance measurements after 25 minutes, the corresponding model parameters were found to be $\mathrm{a}=0.7709, \mathrm{~b}=1447$ and $\mathrm{c}=0.004163$ with $\mathrm{R}^{2}=0.9996$ (Figure S3). 

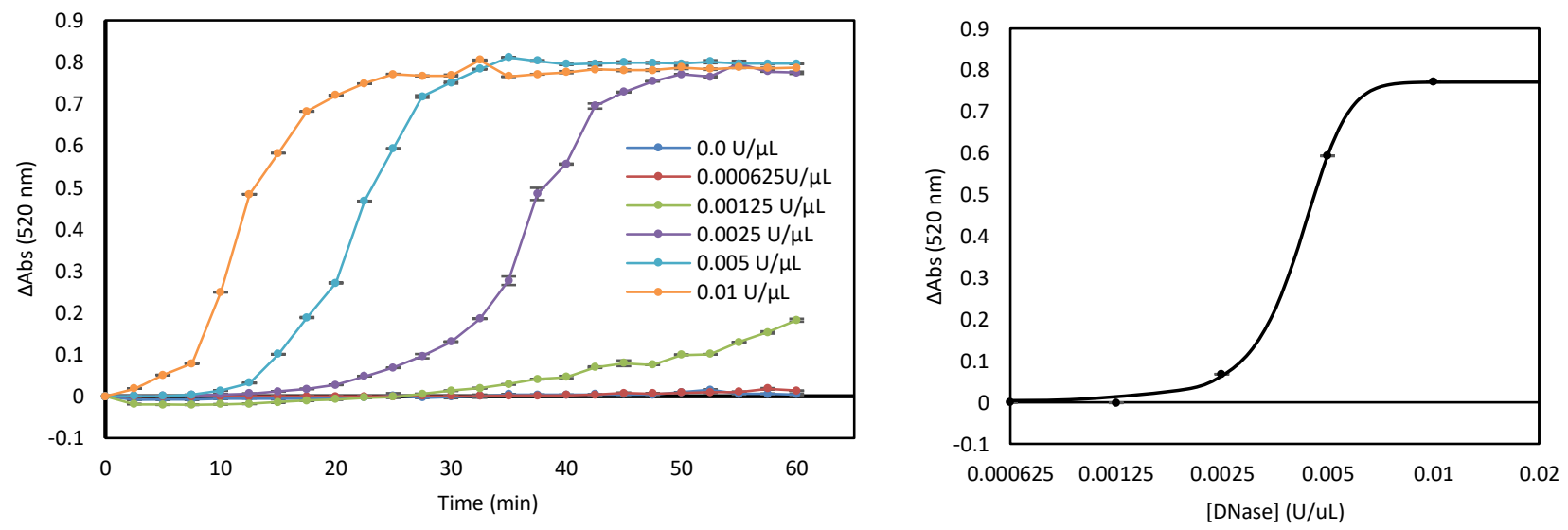

\begin{abstract}
Figure S3. Absorbance of DNaseI sample solutions ranging from $6.25 \times 10^{-4}$ to $0.01 \mathrm{U} / \mu \mathrm{L}$. A) Absorbance of different concentrations of DNaseI plotted against time. B) Absorbance data points at 25 minutes for the different DNaseI concentrations $\left(L L O D=1.26 \times 10^{-3} \mathrm{U} / \mu \mathrm{L}\right.$ and $\left.\mathrm{ULOD}=6.74 \times 10^{-3} \mathrm{U} / \mu \mathrm{L}\right)$. Error bars represent standard error from triplicate experiments.
\end{abstract}

\title{
TETRACYCLINE ASSAY
}

The tetracycline assay described herein was developed as an all-in-one field portable assay for point-of-care testing in resourcelimited settings. ${ }^{4}$ Studies on the individual components of the assay (the cell reporter strips, the X-gal strips and the LB broth tablets) were performed prior to the final all-in-one assay to optimize the components. These studies, as well as the final all-in-one field portable assay are described below.

\section{Materials}

Desalinized pullulan was purchased from Polysciences Inc. (Mn 200,000). Acacia gum (Mn 250,000) was purchased from Millipore Sigma. 5-bromo-4-chloro-3-indolyl- $\beta$-D-galactopyranoside (X-gal) was purchased from Thermo Scientific and prepared at $30 \mathrm{mg} / \mathrm{mL}(73 \mathrm{mM})$ in reagent grade DMSO (Caledon Laboratory Chemicals). Whatman Grade 42 quantitative filter paper was purchased from GE Healthcare. The reporter cells - tetracycline-inducible plasmid pUT-tetlac harboured in E. coli MT102 (pUTtetlac cells) - were a kind gift from Dr. Søren J. Sørensen at the University of Copenhagen. ${ }^{5}$ Unless otherwise stated, all water used in this study was deionized using a Millipore Synthesis A10 purification system to a resistance of 18.2 M $\Omega . c m$. DNA oligonucleonucleotides were purchased from Integrated DNA Technology (IDT), Coralville, USA. All other reagents were of analytical grade and were used as received.

Bacterial Growth and Enumeration

Cultures of the pUT-tetlac cells were grown overnight in LB media supplemented with $100 \mu \mathrm{g} / \mathrm{mL}$ ampicillin (Ap) and $50 \mu \mathrm{g} / \mathrm{mL}$ kanamycin $(\mathrm{Kn})$ in a shaking incubator operated at $37{ }^{\circ} \mathrm{C}$ and $250 \mathrm{rpm}$. Bacterial cell viability was assessed using standard enumeration techniques. Fresh pUT-tetlac cell cultures were serially diluted in PBS, while cells dried on paper sensing strips (see following section) were first liberated by dissolving the sugar matrix in PBS using a vortex mixer then serially diluted in PBS. Dilutions were spread onto $\mathrm{LB}+\mathrm{Ap}+\mathrm{Kn}$ agar plates and incubated at $37^{\circ} \mathrm{C}$. Colonies were counted after 18 hours and cell numbers $(\mathrm{cfu} / \mathrm{ml})$ were calculated $[\mathrm{cfu} / \mathrm{ml}=\#$ colonies $/$ (dilution $\mathrm{x}$ volume plated)].

Preparation of Sugar Entrapped Assay Components

Acacia gum solutions (15\% w/v) were prepared in Milli-Q water, filtered through a Buchner funnel lined with two coffee filters then autoclaved $\left(121^{\circ} \mathrm{C}, 15\right.$ minutes). Pullulan solutions $(7 \% \mathrm{w} / \mathrm{v})$ were prepared similarly but were not filtered. Whatman 42 paper was cut to size and autoclaved $\left(121^{\circ} \mathrm{C}, 20\right.$ minutes) prior to use. Overnight cultures of pUT-tetlac cells (OD600 0.7) were centrifuged (4800x g, $10 \mathrm{~min})$ and the pellets were resuspended in $15 \% \mathrm{w} / \mathrm{v}$ acacia gum or $7 \% \mathrm{w} / \mathrm{v}$ pullulan, concentrating the cells 50 -fold. Whatman 42 paper $(1 \times 0.5 \mathrm{~cm})$ was soaked in $15 \% \mathrm{w} / \mathrm{v}$ acacia gum or $7 \% \mathrm{w} / \mathrm{v}$ pullulan and placed onto parafilm. Concentrated cell suspension $(10 \mu \mathrm{L})$ prepared in the same polymer was added to the paper strips. Paper strips were dried under sterile conditions in a laminar flow hood (1.5 hours) to produce dried reporter cell strips.

LB tablets were prepared by dissolving $10 \mathrm{~g}$ of solid pullulan in $100 \mathrm{~mL}$ of $7.5 \mathrm{x}$ concentrated LB media (7.5 g Bacto-Tryptone, $3.75 \mathrm{~g}$ Bacto-Yeast Extract, $7.5 \mathrm{~g} \mathrm{NaCl})$ then autoclaving $\left(121^{\circ} \mathrm{C}, 20 \mathrm{~min}\right)$. Fifty microliter droplets of the pullulan:LB mixture were pipetted 
onto a parafilm-lined culture dish and allowed to dry in a $37^{\circ} \mathrm{C}$ static air incubator overnight to form solid pullulan tablets. Final reagent concentrations were $10 \% \mathrm{w} / \mathrm{v}$ pullulan containing $7.5 \mathrm{x}$ LB media.

The X-gal solution used to prepare dried substrate strips was prepared by diluting the $30 \mathrm{mg} / \mathrm{ml}$ (73 mM) stock solution of X-gal in DMSO with $10 \% \mathrm{w} / \mathrm{v}$ sterile pullulan to attain final concentrations of $8.5 \mathrm{mg} / \mathrm{mL} \mathrm{X-gal}(20.7 \mathrm{mM})$ and $7 \% \mathrm{w} / \mathrm{v}$ pullulan. The strips were prepared by depositing $400 \mu \mathrm{L}$ of this X-gal solution onto sterile pieces of Whatman 42 paper $(4 \times 2 \mathrm{~cm})$ and drying at $37{ }^{\circ} \mathrm{C}$ in a static incubator for two days to fully remove the DMSO. The dried strips were rolled slightly to insert them into the caps of culture tubes.

Reporter Cell Assays in Microwell Plates

Microplate-based assays were initially conducted using pUT-tetlac cells air-dried in pullulan or acacia gum directly in the wells of a clear 96-well plate. The viability of the dried cells in both sugars was evaluated through enumeration by comparison with the cfu/ml of the original fresh culture (Figure S4A). Evaluation of reporter responses was done using either $10 \mu \mathrm{l}$ of fresh 50 -fold concentrated cells or cells resuspended from acacia gum, which were added to $2 \mathrm{~mL}$ of a tetracycline solution at a concentration of $100 \mu \mathrm{g} / \mathrm{L}$ supplemented with $0.4 \mathrm{mg} / \mathrm{ml} \mathrm{X-gal}(1 \mathrm{mM})$ and LB, followed by recording of $610 \mathrm{~nm}$ absorbance values (absorbance 610$)$ of $100 \mu \mathrm{L}$ sample aliquots over a period of 20 hours (Figure S4B). Induction of reporter cells resuspended from acacia gum or pullulan as described above was also compared (Figure S4C). Replicate experiments were performed on separate days.
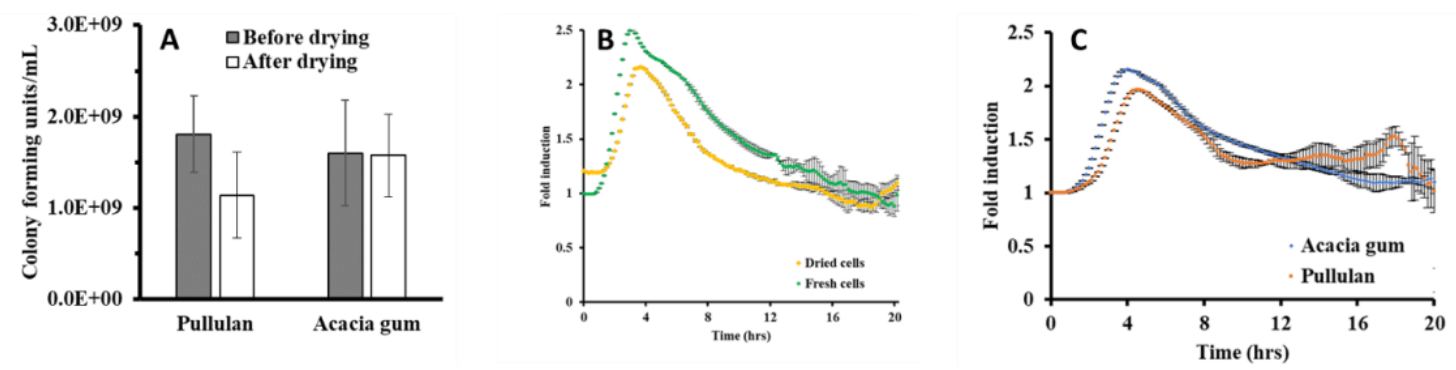

Figure S4. A) Survival of pUT-tetlac reporter cells entrapped in $7 \% \mathrm{w} / \mathrm{v}$ pullulan or $15 \% \mathrm{w} / \mathrm{v}$ acacia gum when dried in a microwell plate. B) Comparison of response to inducer $(100 \mu \mathrm{g} / \mathrm{L}$ tetracycline) of dried cells versus fresh cells over time. C) Comparison of response to inducer $(100 \mu \mathrm{g} / \mathrm{L}$ tetracycline $)$ of dried cells entrapped in pullulan versus acacia gum over time.

Performance of Reporter Cell Paper Strips

The viability of cells dried on paper strips in both pullulan and acacia gum was evaluated through enumeration of cells resus pended from 1-day old paper strips relative to the cell count (in $\mathrm{cfu} / \mathrm{mL}$ ) of the original fresh culture (Figure S5A). The reporter response from 1-day old reporter cell paper strips produced with 15\% w/v acacia gum was also assessed relative to fresh cells (Figure S5B). Fresh cell assays were done as outlined above, except that absorbance 610 measurements were taken after 2 hours of incubation. Reporter cells on paper strips were added to $14 \mathrm{ml}$ polypropylene snap cap tubes (Falcon 352059) tubes containing 2 $\mathrm{mL}$ of tetracycline solutions at concentrations of $0-75 \mu \mathrm{g} / \mathrm{L}$ that were supplemented with X-gal and LB as above, followed by a 2hour incubation time after which $100 \mu \mathrm{L}$ of each sample was transferred to a clear microwell plate to measure the absorbance 610 . Replicate experiments were performed on separate days.
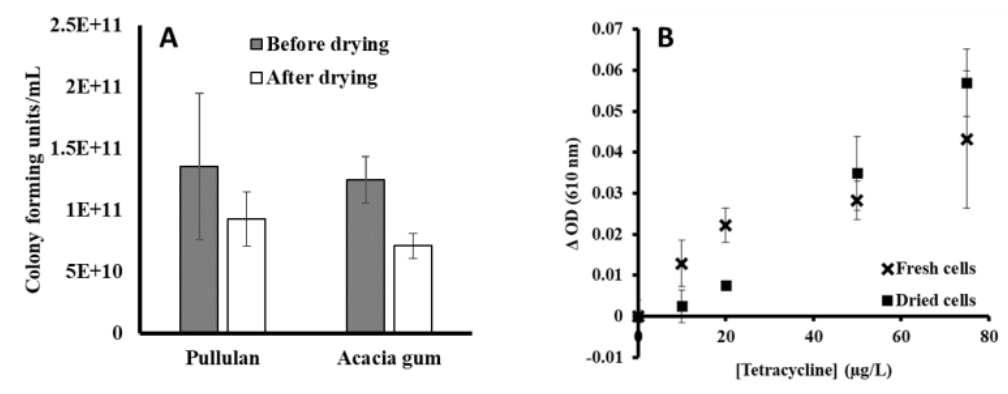

Figure S5. A) Survival of pUT-tetlac reporter cells after drying on paper using acacia gum and pullulan B) pUT-tetlac cell response in MilliQ water spiked with varying tetracycline concentrations in the all-in-one tube assay using fresh and dried cell suspension. Error bars represent standard deviation derived from triplicate experiments. 
Performance of Entrapped X-Gal

As part of developing a field portable assay, the performance of X-gal entrapped in pullulan coated paper strips was also evaluated relative to assays using liquid X-gal (Figure S6). For these assays, fresh reporter cells in LB media were incubated for 1 hour in varying concentrations of tetracycline $(0-75 \mu \mathrm{g} / \mathrm{L})$ followed by addition of either an X-gal coated paper strip or liquid X-gal to provide a final concentration of $1.6 \mathrm{mg} / \mathrm{mL} \mathrm{X-gal}(4 \mathrm{mM})$. The solutions were incubated a further hour, after which $100 \mu \mathrm{L}$ of the samples were transferred to a clear microwell plate, followed by measurement of absorbance at $610 \mathrm{~nm}$. it was observed that the use of the X-gal strip in place of liquid X-gal offered greater sensitivity and a faster response time. If the reaction was left to proceed for a sufficiently long time, it would be expected that both the paper and liquidX-gal tests would reach the same absorbance endpoint at which all X-gal is completely hydrolyzed.

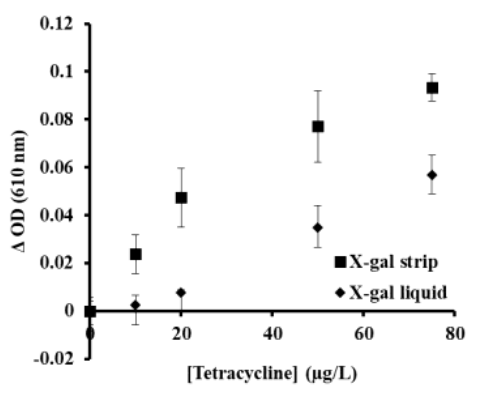

\begin{abstract}
Figure S6. pUT-tetlac cell response using liquid X-gal and dried X-gal strips, in MilliQ water spiked with varying tetracycline concentrations. Absorbance measurements taken 1 hour following X-gal strip addition, and 1.5 hours following X-gal liquid addition.
\end{abstract}

Field Portable Assays in Milli-Q and Lake Water

The final field-portable assay system using reporter cell paper strips (15\% w/v acacia gum), LB tablets (10\% w/v pullulan) and Xgal paper strips $(7 \% \mathrm{w} / \mathrm{v}$ pullulan) was performed on both Milli-Q water and lake water samples that were spiked with varying concentrations of tetracycline $(0-200 \mu \mathrm{g} / \mathrm{L})$. Lake water samples were collected in late summer using clean, unused spring water bottles ( $500 \mathrm{~mL}$ volume). Bottles were rinsed once with lake water then the final sample was collected (total of $1 \mathrm{~L}$ ). Samples were stored in a refrigerator for a maximum of 2 months but were typically tested with a few days of collection. Tetracycline (5 mg/ml stock in ethanol) was spiked into water samples (Milli-Q water or lake water) to produce samples with tetracycline spanning the concentration range noted above. Samples were adjusted to $\mathrm{pH} 6.5$ prior to testing.

The field portable assay (Figure 1 in the main body of the paper) was performed as follows. Tubes containing $2 \mathrm{~mL}$ of sample first had a pullulan tablet with LB added, followed by shaking for 2 minutes to fully dissolve the media. Dried reporter cell strips prepared with $15 \% \mathrm{w} / \mathrm{v}$ acacia gum were then added, mixed for 20 seconds, and then incubated at room temperature for 1 hour. At this point, the sample tube was inverted to introduce the dried X-gal strip (substrate) to the assay. The samples were incubated at room temperature for an additional hour. Samples were either transferred to a clear microwell plate (100 $\mu \mathrm{L}$ aliquots) for triplicate absorbance 610 measurements, or to the custom acetal plate $\left(1 \mathrm{~mL}\right.$ aliquots) followed by affixing the Nix ${ }^{\mathrm{TM}}$ colour sensor over each well to obtain a colour reading, followed by transfer of data to a smartphone to obtain CIELAB coordinates via the Nix ${ }^{\mathrm{TM}}$ colour sensor app. The absorbance 610 or L-coordinate data was plotted against tetracycline concentration to generate calibration curves in Milli- $Q$ and lake water. Field Portable assays were also performed as above using reporter cells entrapped on pullulan coated paper, with data obtained using absorbance 610 measurements for direct comparison to reporter cell strips based on acacia gum.

It was observed that the absorbance values derived from the Nix ${ }^{\mathrm{TM}}$ CIELAB measurements at 1 hour appeared to follow first order kinetics. Assuming that the cellular $\beta$-Galactosidase concentration is proportional to the tetracycline concentration, as shown in prior studies, 5 it can therefore be assumed that the rate of product formation (in other words, the cleavage of X-gal into 5-bromo-4-chloroindoxyl which then forms the characteristic blue 5,5'-dibromo-4,4'-dichloro-indigo), is proportional to both the enzyme concentration as well as the concentration of X-Gal remaining in solution. Most generally, this can be written as follows:

$$
\frac{d[P]}{d t}=\alpha[E] C_{0}-[P]
$$

where $[\mathrm{E}]$ is the $\beta$-Gal enzyme concentration, $\mathrm{C}_{0}$ is the initial $\mathrm{X}$-Gal concentration, $[\mathrm{P}]$ is the indigo product concentration, and $\alpha$ and $\beta$ are constants. In this case, the concentration of enzyme is assumed proportional to the concentration of tetracycline, so the differential equation may be re-written as: 


$$
\frac{d[P]}{d t}=\alpha[\text { Tet }]\left(C_{0}-\beta[P]\right)
$$

Since the concentration of tetracycline (and therefore $\beta$-Gal enzyme) is assumed constant in a given solution, the product formation over time can be given by:

$$
\begin{gathered}
\frac{d[P]}{\left(C_{0}-\beta[P]\right)}=\alpha[\text { Tet }] d t \\
\int_{0}^{[P(t)]} \frac{d[P]}{\left(C_{0}-\beta[P]\right)}=\int_{0}^{t} \alpha[\text { Tet }] d \tau \\
\ln \left(\frac{C_{0}-\beta[P(t)]}{C_{0}}\right)=-\alpha \beta[\text { Tet }] t \\
{[P(t)]=\frac{C_{0}}{\beta}\left(1-e^{-\alpha \beta[\text { Tet }] t}\right)}
\end{gathered}
$$

Therefore, for a specific time, the amount of indigo product formed can be related to the tetracycline concentration according to:

$$
[P(T e t)]=A\left(1-e^{-B[T e t]}\right)
$$

Where constants A, B > 0. According to the Beer-Lambert law, absorbance at the characteristic chromophore maximum absorbance wavelength $\left(\lambda_{\max }\right)$ is proportional to chromophore concentration, thereby suggesting that the following first-order model (Equation S12) could be used to relate absorbance to tetracycline concentration:

$$
[\operatorname{Abs}(\text { Tet })]=A\left(1-e^{-B[\text { Tet }]}\right)
$$

Using the least squares error curve fit application in MATLAB, applied to both the $610 \mathrm{~nm}$ absorbance data from the Nix ${ }^{\mathrm{TM}}$ senso $\mathrm{r}$ measurements after 1 hour and from the TECAN M200 Infinite Pro plate reader, the following curve fits (Table S4) were obtained for the combined MilliQ water and lake water data.

Table S4. Tetracycline model parameters (Equation (S12)) for the $\mathrm{Nix}^{\mathrm{TM}}$ color sensor and plate reader data sets.

\begin{tabular}{|l|l|l|l|l|}
\hline \multirow{2}{*}{ Model Equation } & \multicolumn{3}{|c|}{$[$ Abs (Tet) $]=A\left(1-e^{-B[\text { Tet }]}\right)$} \\
\hline \multirow{2}{*}{ Data Source } & \multicolumn{2}{|c|}{ Nix $^{\text {TM }}$ Measurements } & \multicolumn{2}{c|}{ TECAN Infinite 200Pro Plate Reader } \\
\cline { 2 - 5 } & \multicolumn{1}{|c|}{ MilliQ Water } & \multicolumn{1}{|c|}{ Lake Water } & \multicolumn{1}{c|}{ MilliQ Water } & \multicolumn{1}{c|}{ Lake Water } \\
\hline \multirow{2}{*}{ A (95\% confidence bounds) } & 0.1149 & 0.1038 & 0.07959 & 0.1222 \\
& $(0.1086,0.1212)$ & $(0.0975,0.1102)$ & $(0.07097,0.0882)$ & $(0.1049,0.1394)$ \\
\hline \multirow{2}{*}{ B (95\% confidence bounds) } & 0.05302 & 0.03252 & 0.0478 & 0.02277 \\
& $(0.04007,0.06597)$ & $(0.02508,0.03996)$ & $(0.02543,0.07017)$ & $(0.01273,0.03282)$ \\
\hline $\mathrm{R}^{2}$ & 0.9426 & 0.9567 & 0.8271 & 0.8695 \\
\hline
\end{tabular}

In the case of the plate reader absorbance data, the MilliQ water and lake water samples show consistent readings up to $75 \mu \mathrm{g} / \mathrm{L}$ but show significant deviations at higher concentrations. The $\mathrm{Nix}^{\mathrm{TM}}$ measurements show more consistent readings between the lake water and MilliQ water throughout the entire range of tetracycline concentrations assessed, which suggests that this measurement method not only offers a portable form of quantitative analysis but may also offer a more robust analysis tool when considering the initial solution source/composition.

This cell-based tetracycline reporting procedure is applicable not only to assessing bioavailable tetracycline concentrations in water samples but could be extended to the assessment of tetracycline resulting from veterinary drugs in milk and other food products. For milk in particular, the Canadian maximum residue limit is $100 \mu \mathrm{g} / \mathrm{L}$, which just exceeds the approximately $75 \mu \mathrm{g} / \mathrm{L}$ saturation limit for tetracycline concentration quantification according to the data presented above. ${ }^{6}$ In all applications, addition of the microbial biosensor strip and LB pullulan tablet to a liquid sample, followed by 1-hour incubation, addition of the X-Gal strip, and $\mathrm{Nix}^{\mathrm{TM}}$ color measurements at 0 and 60 minutes would allow for approximation of the $610 \mathrm{~nm}$ sample absorbance and therefore 
tetracycline concentration through the model defined above. The simplicity, portability and low cost of such microbial biosensor offers a favourable alternative to the standard HPLC quantification methods. Other biosensor tests exist for the detection of various antibiotics in applications such as dairy production, with shorter incubation times (8 minutes rather than on the order of hours), however this requires the use of refrigerated test strips and an electronic test strip analyzer, which reduces the portability and accessibility of the assay. ${ }^{7}$

While the current study focused only on the pUT-tetlac reporter system for sensing of tetracycline, many microbial biosensor systems exist for a range of analytes, including heavy metals, small molecules and toxins. ${ }^{8-12}$ Hence, the all-in-one assay described herein should be amenable to the design of portable sensors for a range of analytes of both clinical and environmental importance. The use of polysaccharide matrices, and their integration onto paper strips, can produce highly stable and easy to use bioassay systems and the use of a portable colour reader and associated app allows for detection to be done in the field with no need for specialized equipment. Given that pullulan systems have already been shown to be able to stabilize enzymes, DNAzymes and labile small molecule reagents, the reported paper-based assays could significantly improve environmental monitoring in resource-limited settings, and allow multiple applications across various fields such as food safety and clinical diagnostics that require the use of labile (bio)reagents.13-16 This strategy should also decrease the number of steps needed to complete an analysis and the degree of user intervention required for current multi-step assays.

\section{PROOF OF CONCEPT SMARTPHONE APPLICATION DEVELOPMENT}

The proof-of-concept smartphone app was developed using Xcode 10.1 for Apple devices. The proof-of-concept app includes:

Main Page:

Provides options to enter: Calibration mode, Estimation mode or Settings.

Calibration Page (Not developed):

While not developed, it would be ideal to include a calibration page that:

- If provided as a kit, could prompt user to follow kit directions (i.e. take 1-5 sample measurements, where each sample is of known concentration/pH/etc.)

- $\quad$ Could generate a graph to display the calibration results

- Would provide options to save calibration parameters/view previous calibration curves

Estimation Page:

Allows users to select assay type and obtain an analyte property estimate once initial and final color measurements are input. The user can manually enter XYZ color coordinates (however this could be interchanged with RGB coordinates, CIELAB coordinates, etc.) to generate an estimate based on the calibration curves presented in the paper. It would be ideal to combine this app with preexisting colorimeter applications so that a user could simply "scan" the color and skip the manual entry. The user could then save the measurement, take another measurement or return to the main page.

Settings Page (Not developed):

While not developed, it would be ideal to include a calibration page that would allow users to review/export/delete previous saved measurements. 


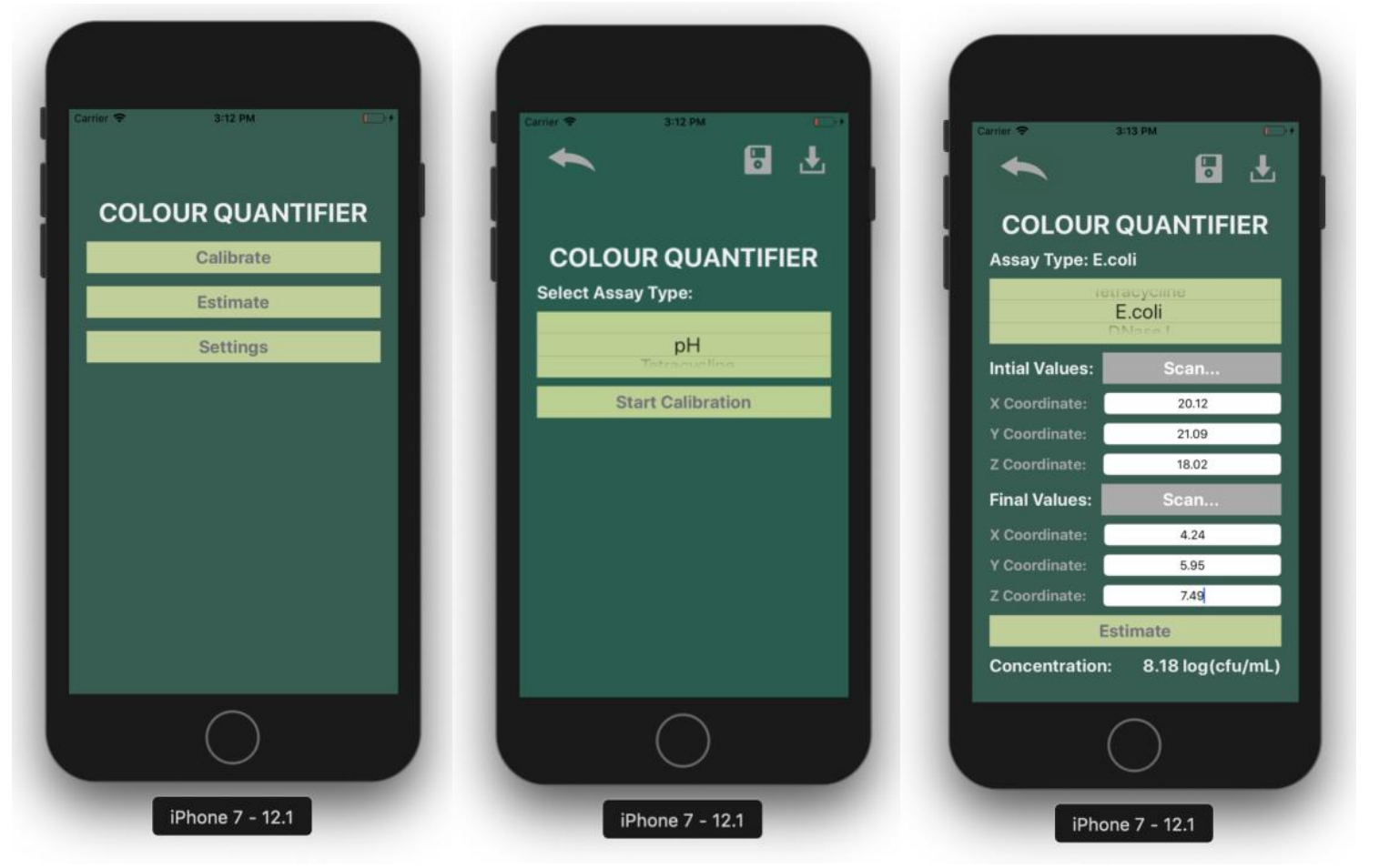

Figure S7. Proof-of-concept smartphone application user interface for colorimetric assays. Left to right: Home page, calibration page and analyte property estimation page. 


\section{References}

1. Burns, S. A. Subtractive Color Mixture Computation. http://scottburns.us/subtractive-color-mixture/.

2. Zhao, W.; Ali, M. M.; Aguirre, S. D.; Brook, M. A.; Li, Y., Paper-Based Bioassays Using Gold Nanoparticle Colorimetric Probes. Anal. Chem. 2008, 80 (22), 8431-8437.

3. Xu, X.; Han, M. S.; Mirkin, C. A., A Gold-Nanoparticle-Based Real-Time Colorimetric Screening Method for Endonuclease Activity and Inhibition. Angew. Chem. Int. Ed. 2007, 46 (19), 3468-3470.

4. Salvo, E. M. The Air-Drying of Eschericha coli Reporters in Natural Polymers and Incorporation into Simple Bioassays. McMaster University, Hamilton, 2017.

5. Hansen, L. H.; Sørensen, S. J., Detection and quantification of tetracyclines by whole cell biosensors. FEMS Microbiol. Lett. 2000, 190 (2), $273-278$.

6. Canada, G. o. List of Maximum Residue Limits (MRLs) for Veterinary Drugs in Foods. https://www.canada.ca/en/health-canada/services/drugshealth-products/veterinary-drugs/maximum-residue-limits-mrls/list-maximum-residue-limits-mrls-veterinary-drugs-foods.html (accessed May 14, 2019).

7. Inc, C. S. Charm Tetracycline Test (TET). http://www.charm.com/products/test-and-kits/antibiotic-tests/rosa-lateral-flow/tet-charmtetracycline-test/ (accessed May 14, 2019).

8. Gopinath, S. C. B.; Tang, T.-H.; Chen, Y.; Citartan, M.; Lakshmipriya, T., Bacterial detection: From microscope to smartphone. Biosens. Bioelectron. 2014, 60, 332-342.

9. Arora, P.; Sindhu, A.; Dilbaghi, N.; Chaudhury, A., Biosensors as innovative tools for the detection of food borne pathogens. Biosens. Bioelectron. 2011, $28(1), 1-12$.

10. Liu, Q.; Wu, C.; Cai, H.; Hu, N.; Zhou, J.; Wang, P., Cell-Based Biosensors and Their Application in Biomedicine. Chem. Rev. 2014, 114 (12), 64236461.

11. Rogers, K. R., Recent advances in biosensor techniques for environmental monitoring. Anal. Chim. Acta 2006, 568 (1), $222-231$.

12. Raut, N.; O'Connor, G.; Pasini, P.; Daunert, S., Engineered cells as biosensing systems in biomedical analysis. Analytical and Bioanalytical Chemistry 2012, 402 (10), 3147-3159.

13. Jahanshahi-Anbuhi, S.; Pennings, K.; Leung, V.; Liu, M.; Carrasquilla, C.; Kannan, B.; Li, Y.; Pelton, R.; Brennan, J. D.; Filipe, C. D. M., Pullulan Encapsulation of Labile Biomolecules to Give Stable Bioassay Tablets. Angew. Chem. Int. Ed. 2014, 53 (24), 6155-6158.

14. Jahanshahi-Anbuhi, S.; Kannan, B.; Leung, V.; Pennings, K.; Liu, M.; Carrasquilla, C.; White, D.; Li, Y.; Pelton, R. H.; Brennan, J. D.; Filipe, C. D. M., Simple and ultrastable all-inclusive pullulan tablets for challenging bioassays. Chemical Science 2016, 7 (3), 2342-2346.

15. Ali, M. M.; Brown, C. L.; Jahanshahi-Anbuhi, S.; Kannan, B.; Li, Y.; Filipe, C. D. M.; Brennan, J. D., A Printed Multicomponent Paper Sensor for Bacterial Detection. Scientific Reports 2017, 7 (1), 12335.

16. Hsieh, P.-Y.; Monsur Ali, M.; Tram, K.; Jahanshahi-Anbuhi, S.; Brown, C. L.; Brennan, J. D.; Filipe, C. D. M.; Li, Y., RNA Protection is Effectively Achieved by Pullulan Film Formation. ChemBioChem 2017, 18 (6), 502-505. 